\title{
Cystic Fibrosis Transmembrane Conductance Regulator Recruitment to Phagosomes in Neutrophils
}

\author{
Yun Zhou $^{\mathrm{a}}$ Kejing Song ${ }^{\mathrm{a}}$ Richard G. Painter ${ }^{\mathrm{a}}$ Martha Aiken $^{\mathrm{a}}$ Jakob Reiser $^{\mathrm{b}}$ \\ Bruce A. Stanton ${ }^{c}$ William M. Nauseef ${ }^{d}$ Guoshun Wang $^{\text {a }}$ \\ ${ }^{a}$ Departments of Microbiology, Genetics and Medicine, Louisiana State University Health Sciences Center, \\ New Orleans, La., 'b Division of Cellular and Gene Therapies, Center for Biologics Evaluation and Research, Food and \\ Drug Administration, Bethesda, Md., 'Departments of Microbiology and Immunology, and Physiology, \\ Dartmouth Medical School, Hanover, N.H., and d Inflammation Program and Department of Medicine, Roy J. and \\ Lucille A. Carver College of Medicine, University of lowa, Coralville, lowa, USA
}

\section{Key Words}

Host defense - Immune response · Neutrophils .

Oxidative burst . Phagocytosis . Hypochlorous acid .

Myeloperoxidase $\cdot$ Chloride channel $\cdot$ Cystic fibrosis

transmembrane conductance regulator

\begin{abstract}
Optimal microbicidal activity of human polymorphonuclear leukocytes (PMN) relies on the generation of toxic agents such as hypochlorous acid ( $\mathrm{HOCl}$ ) in phagosomes. $\mathrm{HOCl}$ formation requires $\mathrm{H}_{2} \mathrm{O}_{2}$ produced by the $\mathrm{NADPH}$ oxidase, myeloperoxidase derived from azurophilic granules, and chloride ion. Chloride transport from cytoplasm into phagosomes requires chloride channels which include cystic fibrosis transmembrane conductance regulator (CFTR), a cAMP-activated chloride channel. However, the phagosomal targeting of CFTR in PMN has not been defined. Using human peripheral blood PMN, we determined that $95-99 \%$ of lysosomal-associated membrane protein 1 (LAMP-1)-positive mature phagosomes were CFTR positive, as judged by
\end{abstract}

immunostaining and flow cytometric analysis. To establish a model cell system to evaluate CFTR phagosomal recruitment, we stably expressed enhanced green fluorescent protein (EGFP) alone, EGFP-wt-CFTR and EGFP- $\Delta$ F508-CFTR fusion proteins in promyelocytic PLB-985 cells, respectively. After differentiation into neutrophil-like cells, CFTR presentation to phagosomes was examined. EGFP-wt-CFTR was observed to associate with phagosomes and colocalize with LAMP-1. Flow cytometric analysis of the isolated phagosomes indicated that such a phagosomal targeting was determined by the CFTR portion of the fusion protein. In contrast, significantly less EGFP- $\Delta$ F508-CFTR was found in phagosomes, indicating a defective targeting of the molecule to the organelle. Importantly, the CFTR corrector compound VRT-325 facilitated the recruitment of $\Delta$ F508-CFTR to phagosomes. These data demonstrate the possibility of pharmacologic correction of impaired recruitment of mutant CFTR, thereby providing a potential means to augment chloride supply to the phagosomes of PMN in patients with cystic fibrosis to enhance their microbicidal function.

Copyright $\odot 2013$ S. Karger AG, Basel

\section{KARGER}

Fax +4161306 1234

E-Mail karger@karger.ch

www.karger.com
(C) 2013 S. Karger AG, Basel

$1662-811 X / 13 / 0053-0219 \$ 38.00 / 0$

Accessible online at:

www.karger.com/jin
Dr. Guoshun Wang

Gene Therapy Program, Departments of Microbiology, Genetics and Medicine Louisiana State University Health Sciences Center

CSRB 642, 533 Bolivar Street, New Orleans, LA 70112 (USA)

E-Mail gwang@lsuhsc.edu 


\section{Introduction}

Cystic fibrosis (CF), one of the most lethal inherited diseases in Caucasians, is caused by mutations of $\mathrm{CF}$ transmembrane conductance regulator (CFTR), a cAMPstimulated chloride channel. Such mutations compromise the normal function of multiple organs and systems including the lung, pancreas, intestines, and vas deferens [1]. Over $90 \%$ of CF patients die of lung disease, which is characterized by chronic bacterial infection, prominent neutrophil infiltration and small airway obstruction [2]. Even though tremendous progress has been made towards the understanding of the pathogenesis of CF and its complications, the key link between the chloride channel defect and the host defense failure has not been definitively established.

CF lung disease is virtually an infection and immunity issue, which involves the interplay between microorganisms and lung host defense. The cellular arm of innate immunity in the lung reflects the combined activities of resident cells, including the pulmonary epithelial cells and macrophages, and of recruited cells, most notably polymorphonuclear leukocytes (PMN). Association of $\mathrm{PMN}$ with CF pathogenesis is recognized due to the fact that they predominate in the patient lung and are the major contributor to lung inflammation and damaging [36]. Disturbance in innate neutrophil function in CF includes excessive recruitment [2], hyperproduction of oxidants [7,8], over-release of degradation enzymes [9] and erroneous delay of apoptosis [10]. Most importantly, PMN are made to kill through compartmentalization of ingested microbes in membrane-bound phagosomes, where toxic oxidants such as hydrogen peroxide $\left(\mathrm{H}_{2} \mathrm{O}_{2}\right)$ and hypochlorous acid ( $\mathrm{HOCl})$ are generated [11-13]. The azurophilic granule protein myeloperoxidase (MPO) catalyzes the oxidation of $\mathrm{Cl}^{-}$to form $\mathrm{HOCl}$, as indicated in the following bioreaction

$$
\mathrm{H}_{2} \mathrm{O}_{2}+\mathrm{Cl}^{-}+\mathrm{H}^{+} \stackrel{\text { MPO }}{\longrightarrow} \mathrm{HOCl}+\mathrm{H}_{2} \mathrm{O}
$$

and availability of this anion in PMN phagosomes is a rate-limiting factor for $\mathrm{HOCl}$ production. Normal PMN express CFTR largely in the membrane of secretory vesicles, and CF PMN or normal PMN pretreated with a CFTR inhibitor or CFTR-depressed neutrophils derived from HL-60 cells via siRNA exhibit reduced $\mathrm{Cl}^{-}$transport into phagosomes and impaired killing of phagocytosed bacteria [14-17]. The data suggest that CFTR serves an important role in normal PMN antimicrobial action. This finding is consistent with the result by reduction in CFTR expression in zebrafish CFTR morphants
[18] and is confirmed by myeloid-specific deletion of CFTR in mice, which leads to a compromised host defense against Pseudomonas aeruginosa infection [19]. Despite the revealing role of CFTR in phagocytic host defense, physical presentation and recruitment of this chloride channel to neutrophil phagosomes has not been clearly defined.

In the current study, we have examined CFTR targeting to the phagosomes of human peripheral blood neutrophils. We have also generated three PLB-985 promyelocytic cell lines expressing enhanced green fluorescent protein (EGFP) alone, EGFP-wt-CFTR or EGFP- $\triangle$ F508CFTR fusion proteins to evaluate the CFTR phagosomal recruitment. Taking advantage of the established cell model, the effect of VRT-325, an agent that repairs the misfolding of $\triangle F 508$ CFTR, on phagosomal recruitment of mutant CFTR has been investigated.

\section{Materials and M§ethods}

\section{Ethics Statement}

The human subject protocol for human peripheral blood withdrawal was approved by the Institutional Review Boards of Louisiana State University Health Sciences Center (New Orleans, La., USA). Written informed consent was obtained from all participants involved in this study.

\section{Chemicals, Reagents and Solutions}

Chloride Ringer's buffer was composed of $122 \mathrm{mM} \mathrm{NaCl}, 20$ mM Hepes, $1.2 \mathrm{mM} \mathrm{MgCl}_{2}, 1.2 \mathrm{mM} \mathrm{CaCl}_{2}, 2.4 \mathrm{mM} \mathrm{K}_{2} \mathrm{HPO}_{4}, 0.6 \mathrm{mM}$ $\mathrm{KH}_{2} \mathrm{PO}_{4}, 10 \mathrm{~mm}$ dextrose, $\mathrm{pH}$ 7.4. Gluconate chloride-free Ringer's buffer was made by substitution of the above chloride salts with equal molar concentration of gluconate salts, except $4 \mathrm{mM}$ calcium gluconate was used to compensate for the mild calcium chelating effect of gluconate. The $\mathrm{KCl}$ relaxation buffer contained $50 \mathrm{~mm} \mathrm{KCl}, 3 \mathrm{~mm} \mathrm{NaCl}, 3.5 \mathrm{~mm} \mathrm{MgCl}_{2}, 0.5 \mathrm{~mm}$ EGTA, $1 \mathrm{~mm}$ ATP, $20 \mathrm{mM}$ PIPES (pH 7.0), $1 \mathrm{~mm}$ PMSF and Sigma protease inhibitor cocktail. Mouse monoclonal anti-human CFTR antibodies 24.1 and 13.1 were obtained from R\&D Systems (Minneapolis, Minn., USA). Alexa Fluor-488-conjugated goat anti-mouse IgG $\left(\mathrm{Fab}^{\prime}\right)_{2}$ fragments were purchased from Invitrogen (Carlsbad, Calif., USA). Cy3-conjugated rabbit anti-human lysosomal-associated membrane protein 1 (LAMP-1) antibody was obtained from Sigma-Aldrich (St. Louis, Mo., USA). The CFTR corrector compound VRT-325 was obtained from the Cystic Fibrosis Foundation. General chemicals were obtained from Sigma-Aldrich.

\section{Lentiviral Vector Construction and Virus Production}

The EGFP-wt-CFTR and EGFP- $\triangle$ F508-CFTR cassettes were released from the original pS65T-EGFP-wt-CFTR and pS65TEGFP- $\triangle$ F508-CFTR plasmids and were subcloned into the pNLEGFP/CMV/WPREdU3 lentiviral transgene plasmid. The resultant constructs (HIV-CMV-EGFP-wt-CFTR and HIV-CMVEGFP- $\triangle$ F508-CFTR) were used to produce the corresponding lentiviral vectors by calcium phosphate triple-plasmid transfection 
using the previously described procedure [20]. The control HIVhUbC-EGFP lentiviral vector, expressing the EGFP transgene under control of the human ubiquitin C promoter, was similarly produced. All viral vectors were pseudotyped with the VSV-G envelope.

\section{Culture and Differentiation of PLB-985 Cells and Lentiviral}

Transduction

The human promyelocytic cell line PLB-985, originally from Dr. Timothy Ley (Washington University, St. Louis, Mo., USA) [21], was cultured in advanced RPMI-1640 (Invitrogen) with $2 \mathrm{mM}$ GlutaMax (Gibco), 10\% heat-inactivated bovine growth serum (Hyclone), $100 \mathrm{U} / \mathrm{ml}$ penicillin, $100 \mu \mathrm{g} / \mathrm{ml}$ streptomycin and 0.25 $\mu \mathrm{g} / \mathrm{ml}$ amphotericin B. For lentiviral transduction, cells in suspension were exposed to HIV-hUbC-EGFP, HIV-CMV-EGFP-wtCFTR and HIV-CMV-EGFP- $\triangle$ F508-CFTR viruses, respectively, at a multiplicity of infection of 20. After stable expression was achieved, the cells were sorted consecutively three times by FACS to obtain the pure population of PLB-985-EGFP, PLB-985-EGFPwt-CFTR or PLB-985-EGFP- $\triangle$ F508-CFTR cells, which stably expressed the corresponding transgene proteins. Where indicated, the cells were terminally differentiated into neutrophil-like cells by induction with $1.25 \%$ DMSO for 5 days.

Flow Cytometric Evaluation of PLB-985 Cell Differentiation

After treated with $1.25 \%$ DMSO for 5 days, the differentiated PLB cells were fixed in $4 \%$ paraformaldehyde for $10 \mathrm{~min}$, washed twice with cold PBS and then resuspended in PBS with $5 \%$ goat serum at a concentration of $1 \times 10^{6}$ cells $/ \mathrm{ml}$. The cells $\left(1 \times 10^{5}, 100\right.$ $\mu \mathrm{l})$ were transferred to a $5-\mathrm{ml}$ culture tube and mixed with the phycoerythrin-conjugated mouse anti-human CD11b antibody (BD Biosciences, San Jose, Calif., USA) at a 1:100 dilution. After incubation for $1 \mathrm{~h}$ in the dark at room temperature, $400 \mu \mathrm{l}$ of PBS was added and cells were analyzed by flow cytometry.

\section{Neutrophil Isolation}

Peripheral blood neutrophils were isolated from freshly drawn human venous blood. The procedure was published previously [16].

Opsonization of Latex Beads

Latex beads were suspended in PBS with $10 \mathrm{mg} / \mathrm{ml} \mathrm{BSA}$ and slowly rotated overnight at $4^{\circ} \mathrm{C}$. After centrifugation at $5,000 \mathrm{~g}$, the beads were washed 3 times with PBS and resuspended in $0.5 \mathrm{ml}$ PBS plus $0.1 \mathrm{ml}$ rabbit anti-BSA serum (Sigma-Aldrich) and incubated overnight with rotation. After three washes with PBS, the coated beads were resuspended in $0.5 \mathrm{ml}$ PBS containing $5 \mathrm{~mm}$ azide and stored at $4^{\circ} \mathrm{C}$. The beads were opsonized with $\mathrm{C} 3 \mathrm{~b}$ by mixing with $10 \%$ fresh male $\mathrm{AB}$ serum at $37^{\circ} \mathrm{C}$ for $30 \mathrm{~min}$, washed 3 times and then resuspended in gluconate Ringer's buffer just prior to use.

\section{Immunofluorescent Staining of Phagosomes from Human}

Peripheral Blood Neutrophils and Flow Cytometry Analysis of CFTR Expression

For this experiment, 2- $\mu \mathrm{m}$ latex microspheres were used. The beads $\left(3.1 \times 10^{9}\right)$ in $3 \mathrm{ml}$ of PBS were coated similarly as above. Human peripheral blood neutrophils $\left(1 \times 10^{7}\right.$ cells $)$ were mixed with $1 \times 10^{8}$ of the opsonized particles in chloride Ringer's solution with $5 \mathrm{mM}$ azide and incubated for $10 \mathrm{~min}$ at $37^{\circ} \mathrm{C}$ with shaking. Azide inclusion here and hereafter was used to prevent the poten- tial MPO-mediated oxidant production that could bleach GFP fluorescence. The cell-bead mixture, resuspended in $5 \mathrm{ml}$ prewarmed Ringer's solution, was plated into a $35-\mathrm{mm}$ tissue culture dish and incubated for an additional 10 min to allow cell attachment to the surface. After 3 vigorous washes to remove free cells and particles, diisopropylfluorophosphate was added at $5 \mathrm{mM}$ and the dish held on ice for $10 \mathrm{~min}$ to inactivate serine proteases. After washing with cold Ringer's buffer, the plate was rinsed with $5 \mathrm{ml}$ of the chloride relaxation buffer with $1 \mathrm{mM}$ ATP. Finally, $1 \mathrm{ml}$ of the same relaxation buffer containing $10 \mu \mathrm{l}$ of Sigma protease inhibitor cocktail was added. The cells were then harvested by scraping and immediately subjected to nitrogen cavitation (400 psi, 10 min on ice). The cavitate was collected and centrifuged $(400 \mathrm{~g})$ at $4^{\circ} \mathrm{C}$ for 5 min to remove intact cells and nuclei. The bead-rich supernatant was recovered for immunostaining. Aliquots $(20 \mu \mathrm{l})$ of the supernatant were incubated with the primary antibody to CFTR (anti-CFTR Ab-24.1 or anti-CFTR Ab-13.1) at $10 \mu \mathrm{g} / \mathrm{ml}$ (R\&D Systems), or with the corresponding isotype control, $\mathrm{IgG}_{2 \mathrm{a}}$ or $\mathrm{IgG}_{1}$ (Southern Biologicals, Birmingham, Ala., USA), respectively, in the presence of $5 \%$ normal goat serum for $1 \mathrm{~h}$ at ambient temperature. If the particles were to be labeled for LAMP-1, Cy3conjugated rabbit anti-human LAMP-1 was added at a 1:100 dilution at this step also. After washing, the particles were resuspended in $20 \mu$ of a 1:500 dilution of $F\left(a b^{\prime}\right)_{2}$ fragments of the Alexa Fluor-488 conjugate of goat anti-mouse IgG. Preliminary experiments showed that this conjugate did not label beads coated with rabbit IgG, and thus, did not cross-react with rabbit IgG. The particles were washed as above and resuspended in $1 \mathrm{ml}$ of PBS and analyzed immediately by flow cytometry using a BD FACSCalibur fluorescence-activated flow cytometer. The instrument was electronically gated on the clearly distinguishable $2-\mu \mathrm{m}$ bead/phagosome population.

\section{Confocal and Conventional Microscopy}

DMSO-differentiated PLB-EGFP-wt-CFTR cells were resuspended in chloride Ringer's buffer, mixed with serum-opsonized $3-\mu \mathrm{m}$ latex beads at a ratio of $1: 10$ and placed onto $12-\mathrm{mm}$ coverslips in a 24 -well dish. After phagocytosis at $37^{\circ} \mathrm{C}$ for $30 \mathrm{~min}$, the cells were fixed in $4 \%$ paraformaldehyde for $10 \mathrm{~min}$ and washed three times with PBS. Then, the coverslips with attached cells were stained with 4'-6-diamidino-2-phenylindole at $10 \mu \mathrm{g} / \mathrm{ml}$ for 30 min, washed three times with PBS, and mounted on microscopic slides with Prolong Gold antifade mounting reagent (Invitrogen). The cells were examined by confocal microscopy. For LAMP-1 immunostaining, the fixed cells were permeabilized with $0.5 \%$ Triton X-100 in PBS for $10 \mathrm{~min}$, followed by incubation overnight with anti-LAMP-1-Cy3 antibody (Sigma-Aldrich) at a 1:300 dilution. The cells were then washed three times with $0.1 \%$ Triton X-100 PBS for 30 min and mounted similarly for observation. The samples, where indicated, were also observed by conventional light and fluorescence microscopy. Micrographs were taken and quantitatively analyzed using Metamorph image acquisition and analysis software. Fluorescent intensities of various phagosomes were quantitated by analyzing their pixel profiles across the center lines of the ingested beads that were drawn by using the software. More than 20 phagosomes were measured and the average net peak fluorescence of either rim (left and right) of the phagosomes was obtained after subtracting the background fluorescence signal. 

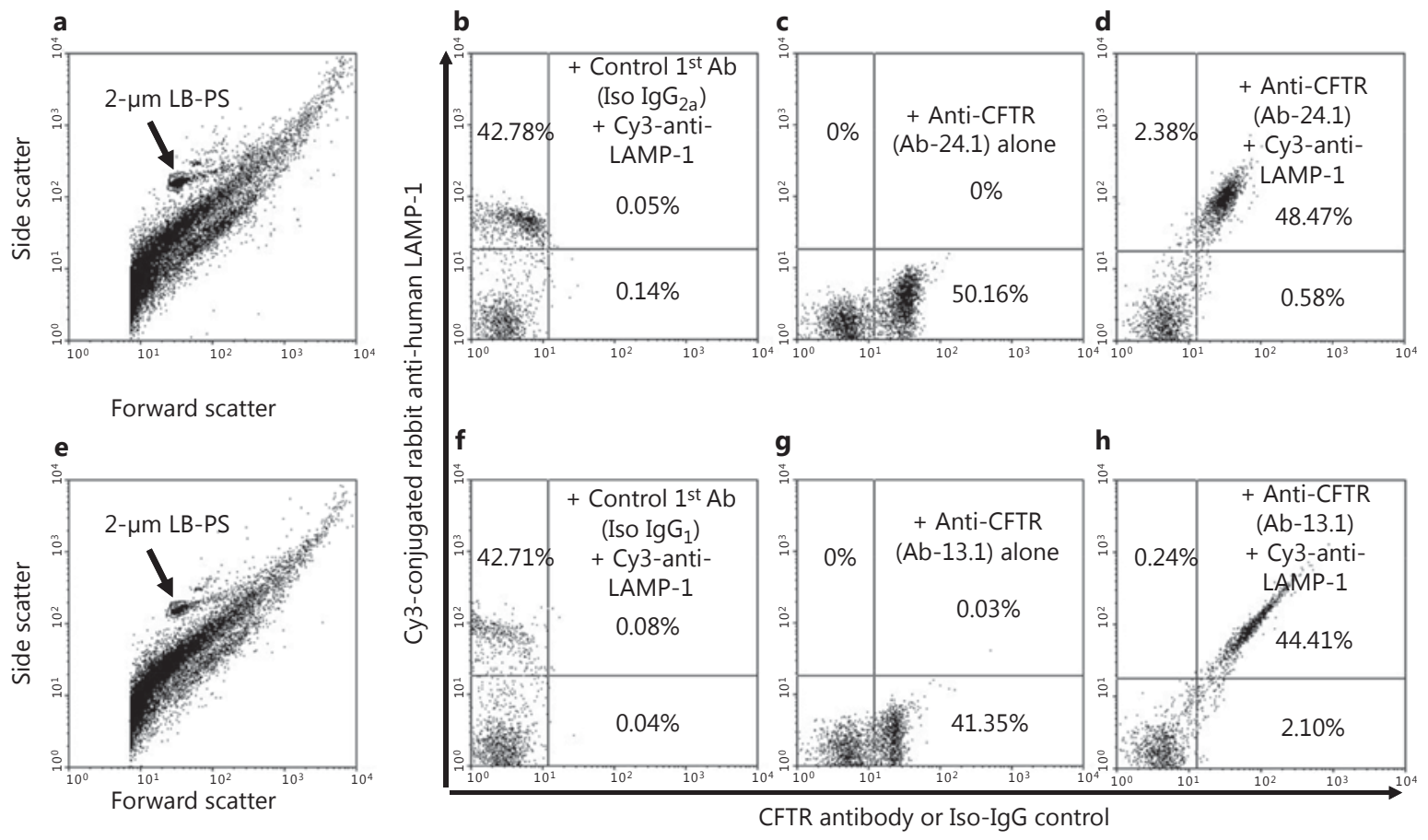

Fig. 1. CFTR expression on phagosomes isolated from peripheral blood human neutrophils. Opsonized 2- $\mu \mathrm{m}$ latex microspheres were fed to isolated human neutrophils for $15 \mathrm{~min}$. After nitrogen cavitation, the cell cavitates were immunostained for CFTR expression using two mouse monoclonal antibodies $(\mathrm{Ab})$ : antiCFTR-24.1 or anti-CFTR-13.1. Where indicated, Cy3 rabbit antihuman LAMP-1 was used to identify late phagosomes. The $2-\mu \mathrm{m}$ monomeric bead/phagosome population was gated by its precise light scattering properties (a, e, arrows). Immunostaining with an-
ti-CFTR antibodies 24.1 or 13.1 alone was performed $(\mathbf{c}, \mathbf{g})$ and compared to their corresponding isotype (Iso) antibody-negative controls $(\mathbf{b}, \mathbf{f})$. Two-color staining with anti-CFTR-24.1 and Cy3LAMP-1 (d) or anti-CFTR-13.1 and Cy3-LAMP-1 (h) shows that most of the CFTR-positive staining was associated with LAMP1-positive phagosomes. The percentages of particles in each quadrant of the dot plots are shown as indicated. LB-PS = Latex beadladen phagosomes.
Flow Cytometric Analysis of CFTR Mobilization to Phagosomes Subsequent to Treatment with the CFTR Corrector Compound VRT-325

PLB-985 parental cells, PLB-985-EGFP-wt-CFTR cells and PLB-985-EGFP- $\triangle$ F508-CFTR cells were differentiated by induction with $1.25 \%$ DMSO for 5 days. For drug treatment, VRT-325 at 10 or $25 \mu \mathrm{M}$ was applied for $10 \mathrm{~h}$ and replenished for another $10 \mathrm{~h}$. Then the cells were spun down at $100 \mathrm{~g}$ for $5 \mathrm{~min}$ and the pellet was resuspended in chloride Ringer's buffer at $1 \times 10^{7}$ cells per milliliter. After diisopropylfluorophosphate $(1 \mathrm{mg} / \mathrm{ml})$ treatment for $10 \mathrm{~min}$, the cells were washed, mixed with preopsonized $3-\mu \mathrm{m}$ latex beads $\left(1 \times 10^{8}\right)$ and incubated on ice for $10 \mathrm{~min}$, followed by phagocytosis in a $100-\mathrm{mm}$ tissue culture dish for $30 \mathrm{~min}$ at $37^{\circ} \mathrm{C}$. After washing away the free beads and nonattached cells with chloride Ringer's buffer, the cells were lysed with $1 \mathrm{ml}$ of the $\mathrm{KCl}$ relaxation buffer and harvested with a policeman rubber. Then, the cells were homogenized by passing through a 25-gauge syringe needle 15 times followed by a 30 -gauge needle for 5 times.
The samples were centrifuged at $100 \mathrm{~g}$ for $5 \mathrm{~min}$ to remove the unbroken cells and nuclei. The supernatants were analyzed by flow cytometry.

\section{Results}

\section{CFTR Expression in Neutrophil Phagosomes}

To establish and validate a quantitative method for the measurement of CFTR expression in phagosomes, we fed peripheral blood neutrophils serum-opsonized $2-\mu \mathrm{m}$ latex beads and immunostained the $\mathrm{N}_{2}$-cavitated cells with either CFTR-specific monoclonal antibody Ab-24.1 or Ab-13.1, in combination with an antibody specific for LAMP-1. The three antibodies recognize cytoplasmic epitopes of the corresponding proteins. Thus, when 
phagosomes were isolated, the recognizable C-terminal domains were exposed and no permeabilization procedure was required. Following immunostaining, the samples were subjected to flow cytometric analysis. Because the phagocytosed latex beads were uniformly spherical in shape with a $2-\mu \mathrm{m}$ diameter, we gated the $2-\mu \mathrm{m}$ bead/ phagosome population (fig. 1a, e, arrows). About $43 \%$ of the phagosomes were LAMP-1 positive (fig. $1 \mathrm{~b}, \mathrm{f}$ ). The CFTR immunostaining showed that approximately $50 \%$ of the phagosomes were positive by using the CFTRAb-24.1 antibody (fig. 1c) and approximately $41 \%$ were positive by using the CFTR Ab-13.1 antibody (fig. 1g). The CFTR and LAMP-1 double-positive phagosomes accounted for $44-48 \%$ of the total phagosome population (fig. 1d, h). Thus, almost all the LAMP-1-positive phagosomes were also CFTR positive. Because LAMP-1 is a protein marker for late and mature phagosomes, these results strongly suggest that CFTR was present on the mature phagosomal membrane which could be quantified by a simple flow cytometric analysis.

\section{PLB-985-Derived Neutrophils Expressing}

EGFP-wt-CFTR or EGFP- $\triangle F 508-C F T R$

Neutrophils are terminally differentiated cells and are not amenable to genetic manipulation. This property limits the possibility of investigating CFTR phagosomal targeting in vitro. To circumvent this limitation, we used the human promyelocytic cell line PLB-985 cells as our experimental model. PLB-985 cells can be readily differentiated into neutrophil-like cells upon treatment with DMSO, yielding cells that mimic the properties of normal neutrophils, including the ability to ingest opsonized particles and activate the NADPH oxidase. To obtain the PLB cells with stable expression of EGFP-wt-CFTR or EGFP- $\triangle$ F508-CFTR protein, two lentiviral vectors were generated and produced. The structures of the two transgene constructs are displayed (fig. $2 \mathrm{a}, \mathrm{b}$ ). The GFP was fused to the N-terminus of wild-type or mutant CFTR. Such CFTR fusion proteins were previously shown to retain the same subcellular localization and function in epithelial cells as does untagged CFTR [22-24]. High-titer lentiviral vectors expressing either the wild-type or mutant CFTR fusion protein were produced and used to transduce PLB-985 cells. After stable expression was achieved, the cultures were sorted multiple times to obtain a pure population of the EGFP-positive cells for each by FACS. Before DMSO differentiation, the PLB-985EGFP-wt-CFTR and the PLB-985-EGFP- $\triangle$ F508-CFTR cells were round and nonadherent in chloride Ringer's buffer (fig. 2c, e). In spite of varying fluorescent intensity, about $100 \%$ of the transduced cells expressed the corresponding EGFP fusion proteins (fig. 2d, f). Flow cytometric analysis demonstrated that the two established cell lines had comparable mean channel fluorescent intensity (fig. 2g). Five days after exposure to 1.25\% DMSO, a large portion of the cells had differentiated into neutrophil-like cells, which was reflected by adherence to the plastic substratum in chloride Ringer's buffer (fig. $2 \mathrm{~h}, \mathrm{j}$ ) and were able to phagocytose serum-opsonized latex particles (fig. 3). The fluorescent intensities for the wild-type and mutant CFTR-expressing cell lines after differentiation remained comparable (fig. 21). To evaluate the differentiation efficacy, we immunostained the parental and the two derived cell lines before and after DMSO induction with the antibody against CD11b, a surface marker for neutrophil maturation. Flow cytometry data indicate that the differentiation protocol can give rise to $52-65 \%$ of mature neutrophil-like cells (fig. $2 \mathrm{~m}-\mathrm{r}$ ).

\section{Wild-Type CFTR Localizes to Phagosomes}

To study CFTR subcellular localization during phagocytosis, differentiated PLB-985-EGFP-wt-CFTR cells were fed serum-opsonized $3-\mu \mathrm{m}$ latex beads and observed using confocal microscopy. The rationale of using the $3-\mu \mathrm{m}$ latex beads here was that phagosomes bearing the larger beads are far more distinct from cell debris and other membrane organelles. Further, the larger phagosomal membrane area produces a higher EGFP signal, improving the signal to noise ratio. Rings of EGFP fluorescence surrounded the phagocytosed beads, indicating the association of EGFP-wt-CFTR with the phagosomes (fig. $3 b, c, f$, arrows), and the late phagosome marker LAMP-1 colocalized with EGFP-wt-CFTR (fig. 3e, arrows). To further assess recruitment of CFTR to phagosomes, we directly isolated the phagosomes followed by examination. A significant number of phagosomes were fluorescent by fluorescence microscopy (fig. 3k, l, arrows) and by flow cytometry (fig. $3 \mathrm{~m}, \mathrm{n}$ ), indicating the presence of EGFP-wt-CFTR on the phagosomes. To be certain that the phagosomal presentation of the EGFP fluorescence required CFTR and was not a consequence of direct GFP association with latex beads, we generated an EGFP-expression-only PLB cell line with the lentiviral vector HIV-hUbC-EGFP. Phagosomes isolated from differentiated EGFP-PLB-985 cell-fed latex beads lacked fluorescence, as judged by confocal microscopy (fig. $3 \mathrm{~g}, \mathrm{~h}$ ) and flow cytometry (fig. 3i, j). These data demonstrate that CFTR was responsible for the localization of the EGFP-wt-CFTR fusion protein at the phagosome. 
Fig. 2. PLB-985 cells and their derived neutrophils expressing EGFP-wt-CFTR and EGFP- $\triangle$ F508-CFTR fusion proteins. a, b Structures of lentiviral vectors bearing the EGFP-wt-CFTR or EGFP- $\triangle$ F508CFTR fusion gene. Schematic drawing shows the structures of the two transgene constructs expressing either EGFP-wtCFTR or EGFP- $\triangle$ F508-CFTR. LTR = Long terminal repeat; $\mathrm{CMV}=$ cytomegalovirus promoter. c-I Undifferentiated PLB-985EGFP-wt-CFTR cells (c, d) and PLB-985EGFP- $\triangle$ F508-CFTR cells (e, f) stably expressed the corresponding CFTR fusion proteins $(\mathbf{d}, \mathbf{f})$. Five days after DMSO induction, the cells differentiated into neutrophil-like cells as evidenced in part by their ability to adhere to, and spread on, the substratum $(\mathbf{h}, \mathbf{j})$ compared to the undifferentiated cells (c, e). The relative expression levels of the EGFP-CFTR fusion proteins were profiled by flow cytometry for the undifferentiated $(\mathbf{g})$ and differentiated cells (I). DIC = Differential interference contrast. The red shaded area is the flow cytometric histogram for PLB-985 parental cells. The black solid line represents the fluorescent profile of the EGFP-wt-CFTR cells and the green solid line that of the EGFP- $\triangle$ F508-CFTR cells. $\mathbf{m}-\mathbf{r}$ Flow cytometric analysis of differentiation of the parental PLB-985 cells and the EGFPCFTR-derived neutrophils. PLB-985, PLB985-EGFP-wt-CFTR and PLB-985-EGFP$\triangle$ F508-CFTR cells were treated with $(\mathbf{n}, \mathbf{p}$, $\mathbf{r})$ or without $(\mathbf{m}, \mathbf{o}, \mathbf{q}) 1.25 \%$ DMSO for 5 days. Then the cells were subjected to immunofluorescence staining with the antibody against human CD11b, a mature neutrophil surface marker. Flow cytometry demonstrates that $>50 \%$ of the cells became mature neutrophils using the differentiation protocol. $\mathrm{PE}=$ Phycoerythrin. The colors refer to the online version of the figure.

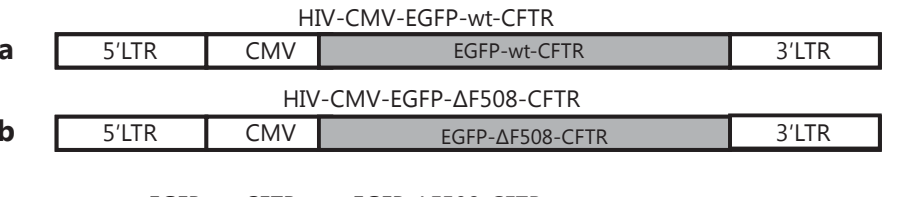

HIV-CMV-EGFP-Wt-CFTR

\begin{tabular}{|c|c|c|c|c|}
\hline $\mathbf{a}$ & $5^{\prime}$ LTR & CMV & EGFP-wt-CFTR & 3'LTR \\
\hline \multicolumn{5}{|c|}{ HIV-CMV-EGFP- $\Delta$ F508-CFTR } \\
\hline b & $5^{\prime}$ LTR & CMV & EGFP- $\triangle$ F508-CFTR & 3'LTR \\
\hline
\end{tabular}

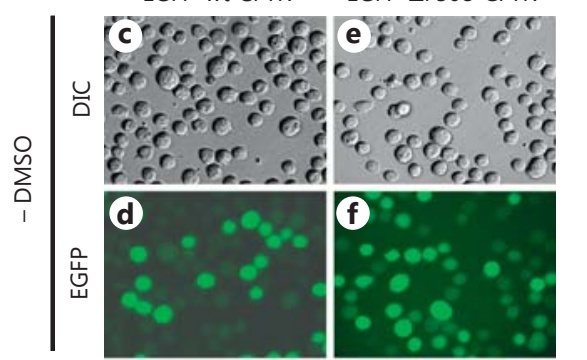

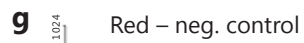

Black - EGFP-wt-CFTR

Green - EGFP- $\triangle$ F508-CFTR
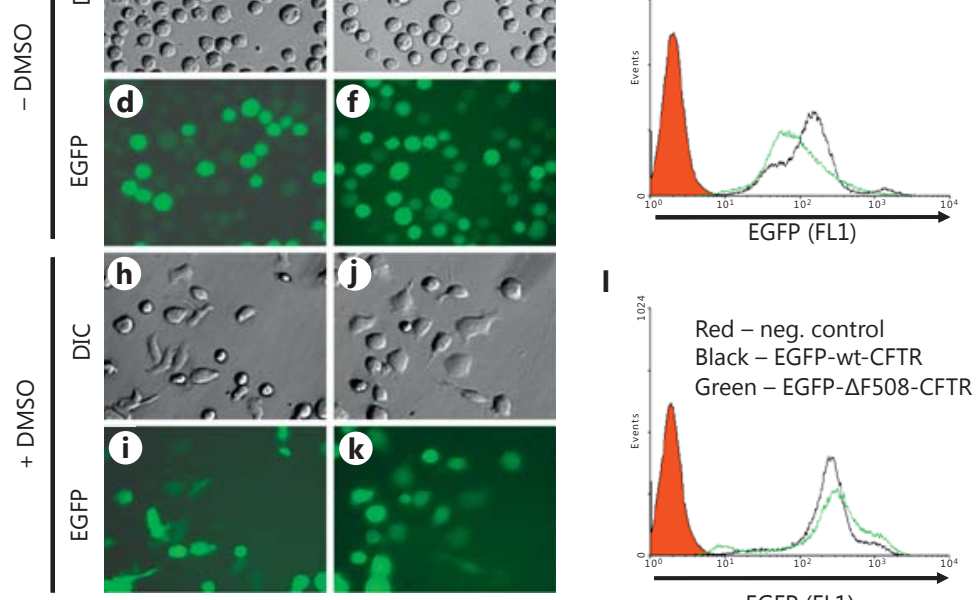

|
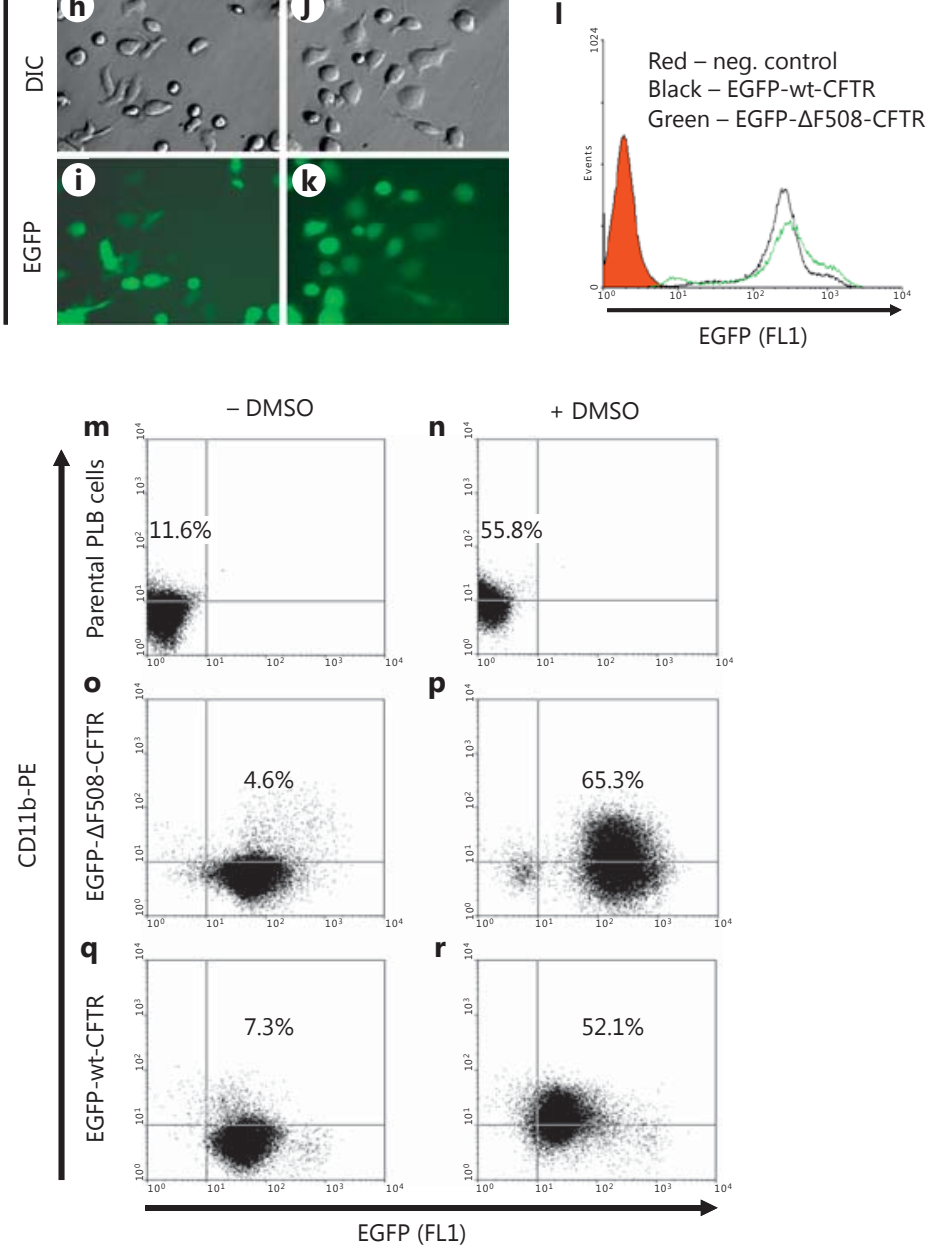

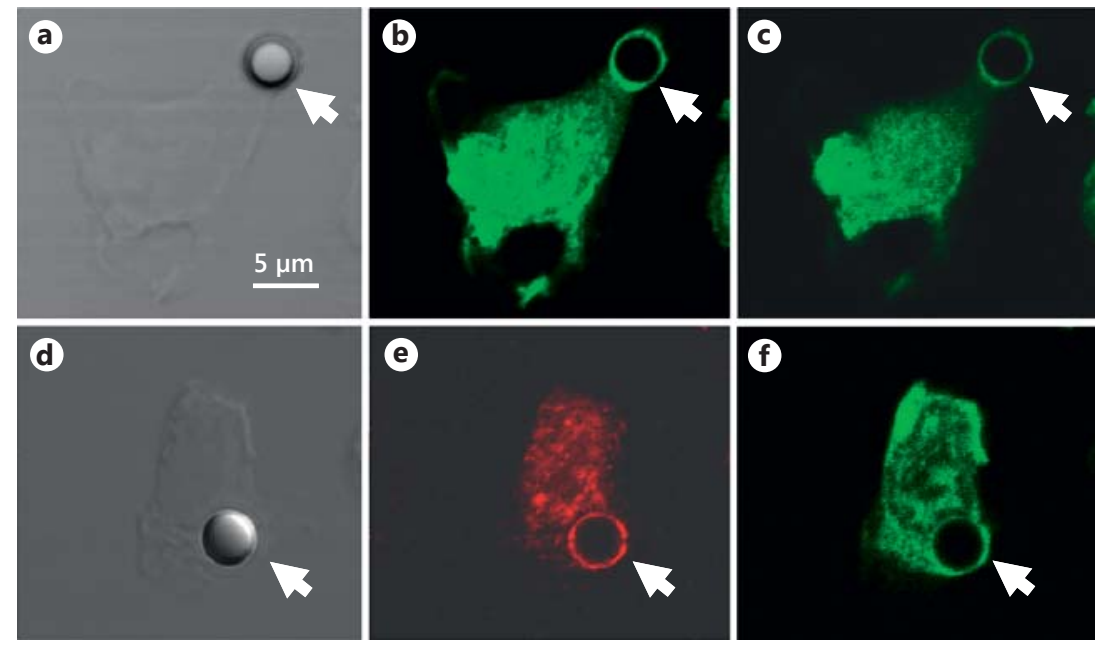

DIC
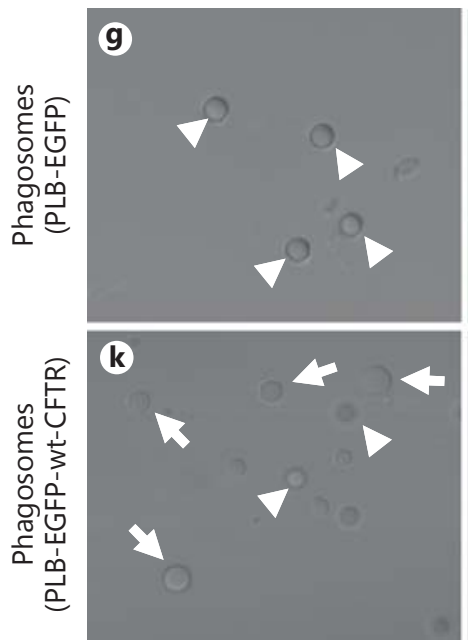

EGFP
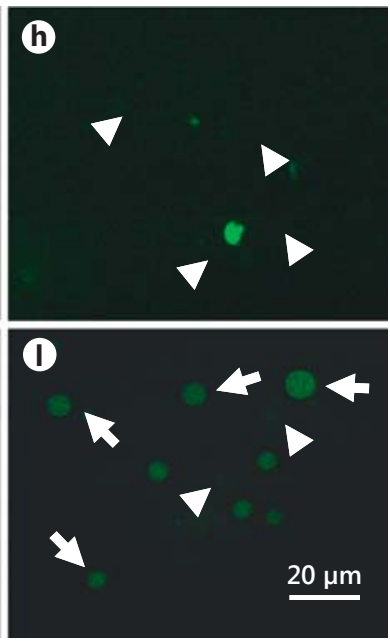

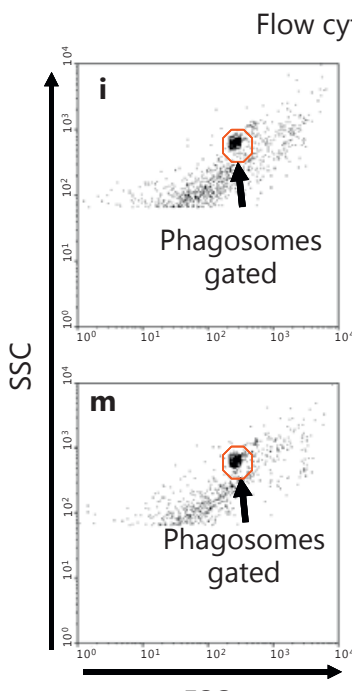

$\mathrm{FSC}$

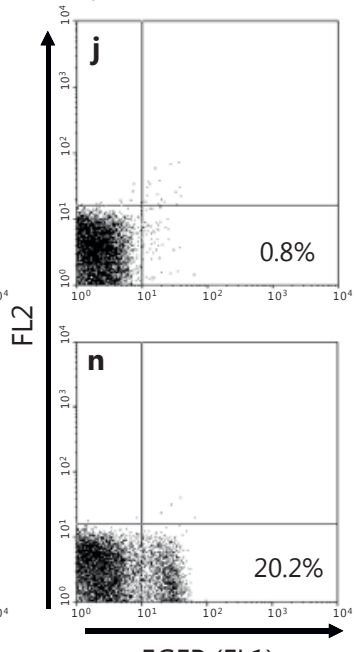

$\operatorname{EGFP}(\mathrm{FL} 1)$
Fig. 3. EGFP-wt-CFTR localizes to phagosomes. PLB-985-EGFPwt-CFTR cells were differentiated into neutrophil-like cells with DMSO for 5 days. After phagocytosis of 3- $\mu \mathrm{m}$ opsonized latex beads, the cells were examined by confocal microscopy. The differential interference contrast image (a) of a cell with an internalized bead and the two X-Y section images from the top to the bottom of the same cell $(\mathbf{b}, \mathbf{c})$ are shown. Arrows point to the bead. To confirm the CFTR-phagosome association, a bead-phagocytosed cell (d) was immunostained for LAMP-1. The result demonstrates a typical phagosomal localization of LAMP-1, a late phagosome marker (e), which colocalized with the EGFP-wt-CFTR (f). Differentiated PLB-985-EGFP and PLB-985-EGFP-wt-CFTR cells were fed serum-opsonized 3- $\mu \mathrm{m}$ latex beads. Phagosomes were isolated and examined by fluorescence microscopy and flow cytometry. EGFP protein alone was not enriched in or targeted to the phagosomes isolated from the PLB-985-EGFP cells. Differential interference contrast (DIC) image (g), fluorescence image (h) and flow cytometry dot plot figures $(\mathbf{i}, \mathbf{j})$ are shown. Arrowheads point to the phagosomes which are negative for EGFP expression. In contrast, EGFP-wt-CFTR was associated with the phagosomes from the PLB-985-EGFP-wt-CFTR cells. Displayed are the differential interference contrast image (k), fluorescence image (I) and the flow cytometry dot plot figures $(\mathbf{m}, \mathbf{n})$. Arrows point to the phagosomes positive for EGFP-wt-CFTR $(\mathbf{k}, \mathbf{I})$. Arrowheads point to the phagosomes negative for EGFP-wt-CFTR. 


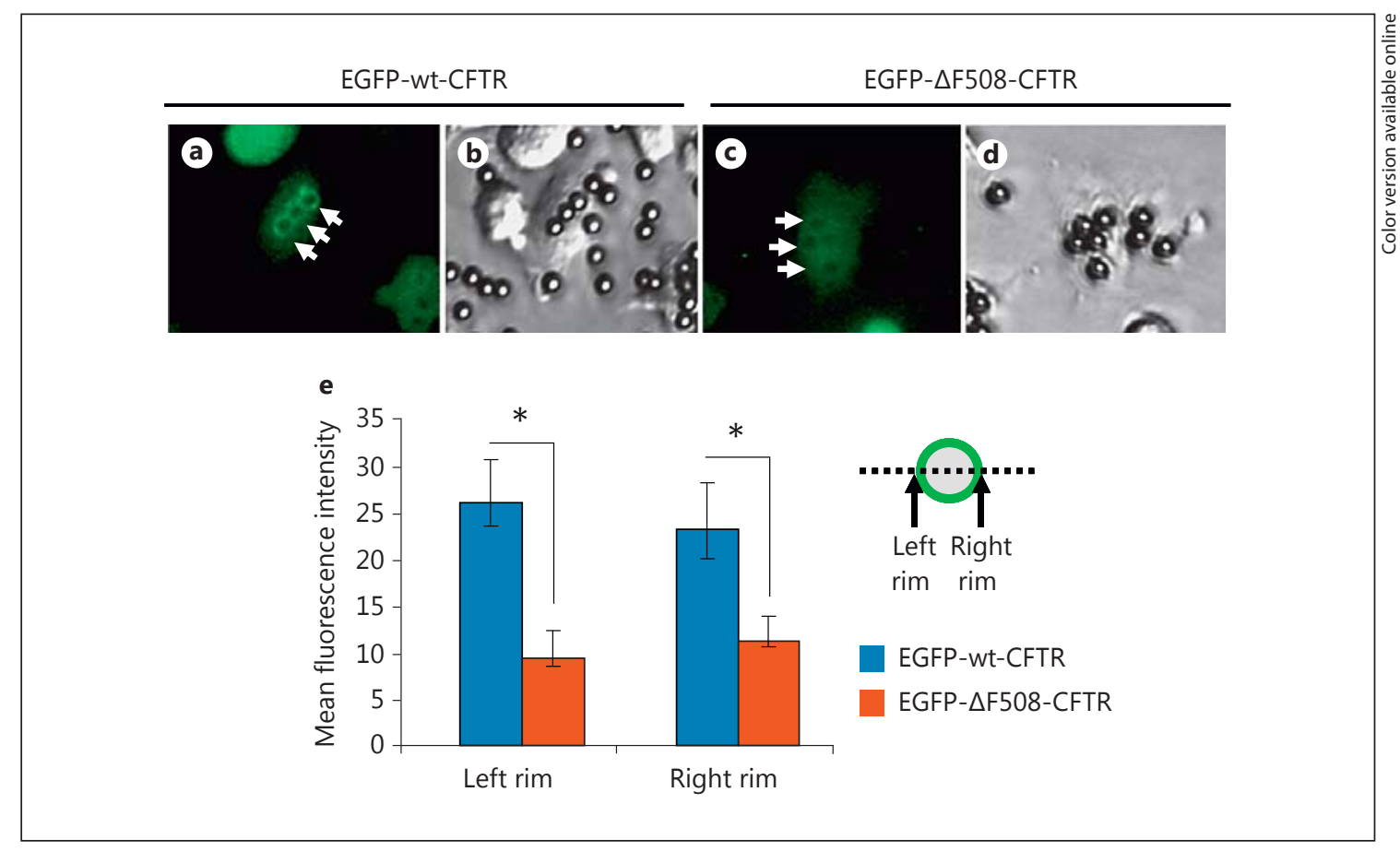

Fig. 4. Significantly less $\Delta$ F508-CFTR fusion protein is associated with phagosomes. The fluorescent (a) and phase-contrast (b) micrographs show EGFP-wt-CFTR association with phagosomes by microscopy. Distinct fluorescent rings are noticeable (a, arrows). Identically prepared cells expressing EGFP- $\Delta$ F508-CFTR had little EGFP fluorescence associated with phagosomes (c, arrows). Quantification of the pixel intensity across the phagosomes was profiled.
The peak fluorescence intensity of either side of the rim of each phagosome was obtained. After subtraction of the background, the mean net peak fluorescence is displayed and compared between the differentiated EGFP-wt-CFTR and EGFP- $\Delta$ F508-CFTR PLB985 cells (e). Statistical analyses show significant differences, as marked with asterisks, by Student's t test $(\mathrm{n}=21$; $\mathrm{p}<0.01)$.

CFTR fusion protein (fig. 4e). This result was confirmed by flow cytometric analysis of the isolated phagosomes (fig. 5b, e).

Enhancement of $\triangle$ F508-CFTR Targeting to Neutrophil Phagosomes in Response to Treatment with the CFTR Corrector Compound VTR-325

The CFTR corrector compound VRT-325 rescues the misfolding defect of $\triangle$ F508-CFTR and facilitates the translocation of $\triangle F 508$-CFTR to the surface of epithelial cells [25]. We hypothesized that VRT-325 would enhance the mutant CFTR phagosome targeting in neutrophils. To test the hypothesis, differentiated PLB-985 cells, both expressing EGFP-wt-CFTR and EGFP$\triangle$ F508-CFTR, were incubated with varied concentrations of VRT-325 $(0,10$ and $25 \mu \mathrm{M})$ for $20 \mathrm{~h}$ prior to phagocytosis of the opsonized 3- $\mu \mathrm{m}$ latex beads. Association of wild-type and mutant CFTR was assessed by flow cytometry of GFP-positive phagosomes (fig. 5). There was no inherent fluorescence of latex bead-con- 

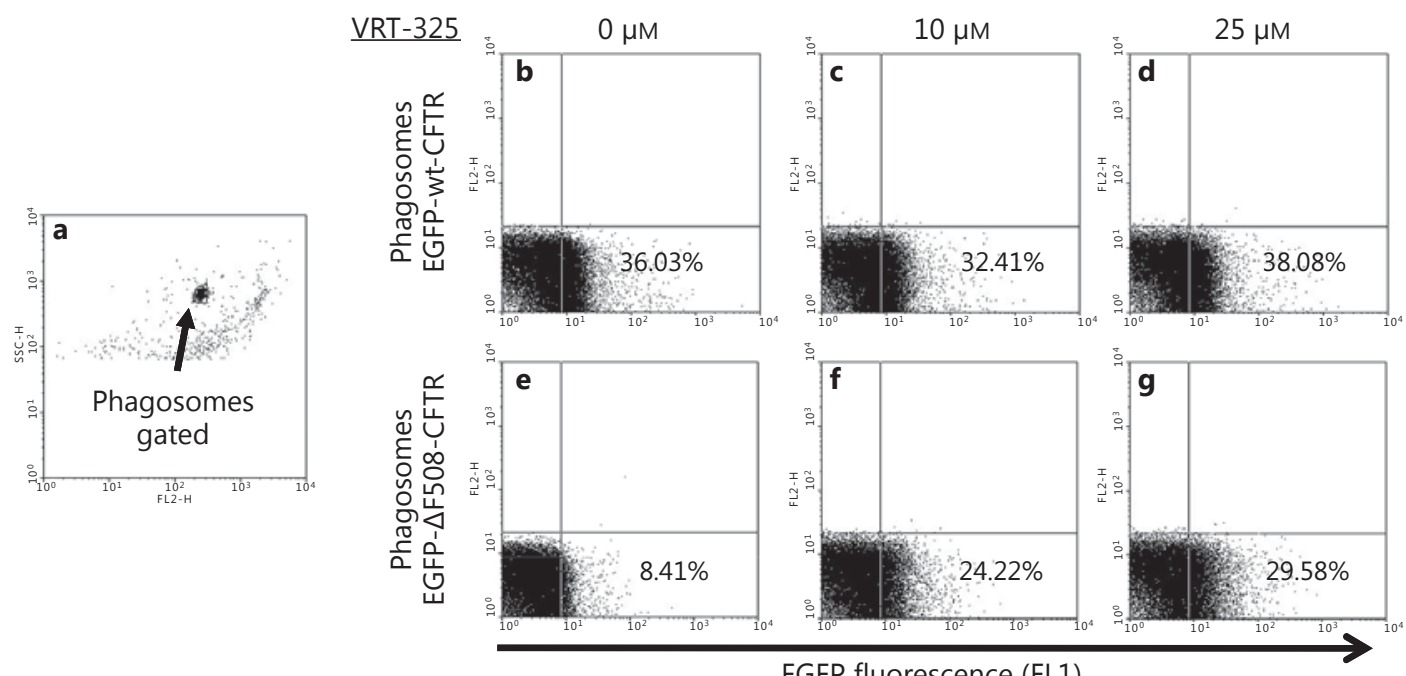

EGFP fluorescence (FL1)

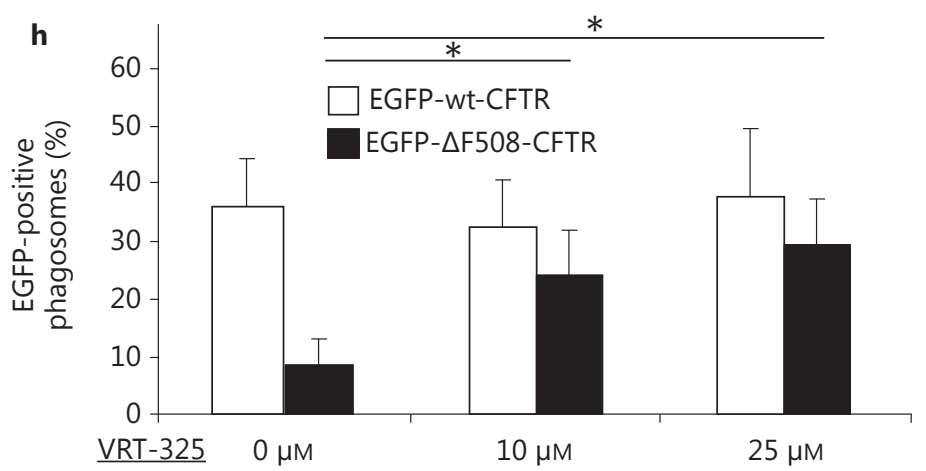

Fig. 5. Enhancement of $\Delta$ F508-CFTR targeting to phagosomes by the CFTR corrector drug VRT-325. Differentiated PLB-985EGFP-wt-CFTR and PLB-985-EGFP- $\Delta$ F508-CFTR cells were treated with 10 or $25 \mu \mathrm{M}$ VRT-325 for $20 \mathrm{~h}$ and were fed the opsonized 3- $\mu \mathrm{m}$ latex beads. After homogenization, the samples were analyzed by flow cytometry to examine the EGFP fluorescence on phagosomes. The representative gating for $3-\mu \mathrm{m}$ phagosomes is displayed (a). VRT-325 treatment did not significantly alter the percent of phagosomes positive for EGFP-wt-CFTR (b-d). However, similar drug treatment significantly increased the presentation of EGFP- $\triangle$ F508-CFTR to the phagosomes $(\mathbf{e}-\mathbf{g})$. Statistical data are displayed (h). Asterisks indicate significant differences by Student's t test $(\mathrm{n}=5 ; \mathrm{p}<0.01)$. taining phagosomes, as phagosomes from nontransfected differentiated PLB-985 cells had no EGFP fluorescence, regardless of VRT-325 drug treatment (data not shown). Without VRT-325 treatment, approximately $36 \%$ of the phagosomes isolated from the EGFP-wtCFTR-differentiated PLB-985 cells were positive for EGFP-wt-CFTR (fig. 5b), whereas only about $8 \%$ of the phagosomes from the EGFP- $\triangle$ F508-CFTR PLB-985 cells were positive for EGFP- $\triangle$ F508-CFTR (fig. 5e). VRT-325 at the concentrations of 10 and $25 \mu \mathrm{M}$ signifi- cantly promoted $\triangle$ F508-CFTR targeting to phagosomes with approximately 1.5 - to 3.5 -fold enhancement as compared to the no-drug control under identical experimental condition (fig. 5e-h). In contrast to its effects on mutant CFTR, VT-325 treatment had a negligible effect on the behavior of wild-type CFTR (fig. 5b-d, h). Furthermore, the mean channel fluorescence intensity for EGFP- $\triangle$ F508-CFTR increased from 20.9 to 43.9, when treated with $10 \mu \mathrm{M}$ VRT-325. The data suggest that VRT-325 enhanced $\triangle$ F508-CFTR targeting to phago- 
somes. Taken together, these results suggest that the CFTR modulator VRT-325 facilitated the trafficking of $\triangle$ F508-CFTR to neutrophil phagosomes, thereby providing the potential to enhance chloride supply to phagosomes of CF neutrophils.

\section{Discussion}

Neutrophils are responsible for the earliest innate immune response to infection, and most of their antimicrobial activity against ingested microbes is confined within phagosomes. However, exuberant neutrophil activation can culminate in extracellular release of oxidants and granule contents that can lead to local damage to normal tissue. Lung infections cause significant morbidity and mortality in patients with CF even in the presence of neutrophil infiltration into infected lungs. Yet despite a successful inflammatory response, neutrophils fail to eradicate invading microbes in the CF lung. Thus, the quality, not the quantity, of the host response to infection appears to be defective in patients with CF. In normal neutrophils, efficient microbicidal action requires $\mathrm{HOCl}$ production, the result of oxidation of chloride anion by MPO in the presence of hydrogen peroxide [26]. Such an $\mathrm{HOCl}$ biosynthesis is pivotal to phagocytic host defense, which is reflected by MPO-deficient humans and mice who are susceptible to certain fungal and bacterial infections [2730]. CF lung disease is characterized by opportunistic bacterial infection such as P. aeruginosa and Staphylococcus aureus. These pathogens do not typically cause infections in healthy subjects. Neutrophils have been demonstrated to be indispensable in cellular innate immunity to eradicate $P$. aeruginosa infection. Neutropenic mice succumb to lung inoculation as few as 100 colony-forming units [31]. We have previously demonstrated that $P$. aeruginosa is more resistant to $\mathrm{HOCl}$ than to other organisms but can be killed by sufficient concentrations of the oxidant in vitro, and CF neutrophils produce less $\mathrm{HOCl}$ $[14,15,32]$. Thus, it is possible that a fully functioning phagosome would efficiently kill $P$. aeruginosa by achieving lethal doses of $\mathrm{HOCl}$, but a chloride-deficient phagosome such as seen in CF patient neutrophils would not produce enough $\mathrm{HOCl}$ to effectively kill this organism. These data altogether suggest that the CFTR chloride channel plays an important role in phagocyte innate immunity, including the normal inflammatory response to pulmonary infections.

Nearly $90 \%$ of the oxygen consumed by the phagocyte $\mathrm{NADPH}$ oxidase during phagocytosis, which is approxi- mately 3-4 nmol $\mathrm{O}_{2}$ per min per million cells $[33,34]$, is converted ultimately to $\mathrm{HOCl}$ [34]. Accordingly, an equivalent amount of chloride must be transported into phagosomes to support $\mathrm{HOCl}$ production. We have measured the rate of chloride uptake directly by living normal neutrophil phagosomes to be in the order of $0.31 \mathrm{mM}$ per second under the condition of a maximally active NADPH oxidase and complete inhibition of MPO with azide [14]. The corresponding luminal concentration of chloride is approximately $70 \mathrm{mM}$ under steady-state conditions [35]. However, in CF neutrophil phagosomes, the chloride influx rate is about $0.043 \pm 0.012 \mathrm{~mm}$ per second and the steady-state chloride concentration is about $25.5 \pm 3.3$ $\mathrm{mm}$ [14]. If we assume that the zymason particles were perfectly round and solid and the gap between the particle surface and the phagosomal membrane was $0.25 \mu \mathrm{m}$, then the steady-state chloride amount in each phagosome within the normal neutrophils would be $0.582 \mathrm{pmol}$ and the initial chloride influx rate $2.576 \mathrm{fmol}$ per second. In contrast, the steady-state chloride amount in each phagosome within the CF neutrophils would be $0.212 \mathrm{pmol}$ and the initial chloride influx rate 0.357 fmol per second. Therefore, CFTR is a major contributor to the chloride influx into phagosomes, especially at the acute phase of chloride supply. Thus, the recruitment of CFTR to phagosomes in their formation is consistent with its importance in phagosome-associated functions.

In this report, we have used flow cytometry to study protein targeting to phagosomes in their isolated state. It was because phagosomes assume the shape of their phagocytosed particulates that we could control and gate the phagosomal population for experimental investigation. The criteria for our judging the gated population to be phagosomes were the following: (1) the bare beads without phagocytosis had the identical light scatter properties, which were distinct from the remaining cell debris and membrane organelles; (2) LAMP-1, a well-recognized marker for mature neutrophil phagosomes, was associated with the gated population; (3) the similarly gated phagosomes could actively produce hydrogen peroxide and $\mathrm{HOCl}$ acid, as reported [36]. Thus, the data suggest that direct flow cytometry of neutrophil homogenates without further phagosome isolation could be developed into a relatively simple and reliable method to study phagosomal properties.

An unexpected finding in the current study is that the CFTR corrector compound VRT-325 significantly enhanced the level of $\triangle F 508$-CFTR on phagosomes. In epithelial cells, $\Delta$ F508-CFTR is not processed correctly and $<5 \%$ of normal levels reach the cell surface membrane 
$[37,38]$. However, once mutant CFTR reaches the cell membrane, it retains some channel function, thereby providing the rationale for searching for pharmaceuticals to correct the defect for therapy $[37,39,40]$. Our data suggest that VRT-325 had analogous effects on biosynthesis and targeting of $\triangle$ F508-CFTR in PLB-985 cells, presumably correcting the folding defect during biosynthesis and thereby promoting proper trafficking of $\triangle$ F508-CFTR to secretory vesicles. Once stored in secretory vesicles, $\triangle$ F508-CFTR was incorporated into phagosomes as secretory vesicles fused with nascent phagosomes during ingestion of particles. Our finding that $\triangle$ F508-CFTR was present at the phagosomal membrane has important clinical implications, as VRT-325 or other effective CFTR corrector drugs may chaperone more $\Delta$ F508-CFTR to the secretory vesicle, eventually providing more CFTR to phagosomes and thereby facilitating more chloride transport and $\mathrm{HOCl}$ production. As the data indicate, VRT325 shows promise in facilitating translocation of the mutant CFTR to neutrophil phagosomes.
In conclusion, we have demonstrated that the CFTR chloride channel was recruited to neutrophil phagosomes. $\triangle$ F508-CFTR failed to target the organelle. Furthermore, the pharmacological CFTR chaperone VRT325 significantly increased $\triangle$ F508-CFTR trafficking to the phagosomes. These findings confirm the importance of CFTR in phagosomal chloride transport, $\mathrm{HOCl}$ production and antimicrobial activity and provide the rationale of pharmacological rescue of the CFTR misfolding in CF PMN.

\section{Acknowledgements}

The authors would like to thank the volunteers who donated their blood and the Cystic Fibrosis Foundation for providing the CFTR corrector compound VRT-325. We also thank Dr. Nick Lanson for his critical reading of the manuscript. This work was supported by a grant from the National Institutes of Health (1R01AI72327) to G.W. B.A.S. was supported by a CF RDP (STANTO07R0) and NIH R01 HL074175.

\section{References}

1 Welsh MJ, Ramsey BW, Accurso F, Cutting G: Cystic fibrosis; in Scriver CR (ed): Metabolic and Molecular Basis of Inherited Disease. New York, McGraw-Hill, 2001, pp 5121-5188.

2 Davis PB, Drumm M, Konstan MW: Cystic fibrosis. Am J Respir Crit Care Med 1996;154: 1229-1256.

3 Witko-Sarsat V, Sermet-Gaudelus I, Lenoir G, Descamps-Latscha B: Inflammation and CFTR: might neutrophils be the key in cystic fibrosis? Mediators Inflamm 1999;8:7-11.

4 Downey DG, Bell SC, Elborn JS: Neutrophils in cystic fibrosis. Thorax 2009;64:81-88.

5 Hayes E, Pohl K, McElvaney NG, Reeves EP: The cystic fibrosis neutrophil: a specialized yet potentially defective cell. Arch Immunol Ther Exp (Warsz) 2011;59:97-112.

6 Ratner D, Mueller C: Immune responses in cystic fibrosis: are they intrinsically defective? Am J Respir Cell Mol Biol 2012;46:715-722.

7 Witko-Sarsat V, Allen RC, Paulais M, Nguyen AT, Bessou G, Lenoir G, Descamps-Latscha B: Disturbed myeloperoxidase-dependent activity of neutrophils in cystic fibrosis homozygotes and heterozygotes, and its correction by amiloride. J Immunol 1996;157:27282735.

8 Witko-Sarsat V, Delacourt C, Rabier D, Bardet J, Nguyen AT, Descamps-Latscha B: Neutrophil-derived long-lived oxidants in cystic fibrosis sputum. Am J Respir Crit Care Med 1995;152:1910-1916.
\$9 Taggart C, Coakley RJ, Greally P, Canny G, O'Neill SJ, McElvaney NG: Increased elastase release by CF neutrophils is mediated by tumor necrosis factor-alpha and interleukin-8. Am J Physiol Lung Cell Mol Physiol 2000; 278:L33-L41.

10 Moriceau S, Lenoir G, Witko-Sarsat V: In cystic fibrosis homozygotes and heterozygotes, neutrophil apoptosis is delayed and modulated by diamide or roscovitine: evidence for an innate neutrophil disturbance. J Innate Immun 2010;2:260-266.

$\checkmark 11$ Hampton MB, Kettle AJ, Winterbourn CC: Inside the neutrophil phagosome: oxidants, myeloperoxidase, and bacterial killing. Blood 1998;92:3007-3017.

12 Klebanoff SJ: Myeloperoxidase: friend and foe. J Leukoc Biol 2005;77:598-625.

13 Nauseef WM: How human neutrophils kill and degrade microbes: an integrated view. Immunol Rev 2007;219:88-102.

14 Painter RG, Marrero L, Lombard GA, Valentine VG, Nauseef WM, Wang G: CFTR-mediated halide transport in phagosomes of human neutrophils. J Leukoc Biol 2010;87:933942.

15 Painter RG, Valentine VG, Lanson NA Jr, Leidal K, Zhang Q, Lombard G, Thompson C, Viswanathan A, Nauseef WM, Wang G: CFTR expression in human neutrophils and the phagolysosomal chlorination defect in cystic fibrosis. Biochemistry 2006;45:1026010269.
16 Painter RG, Bonvillain RW, Valentine VG, Lombard GA, LaPlace SG, Nauseef WM, Wang G: The role of chloride anion and CFTR in killing of Pseudomonas aeruginosa by normal and CF neutrophils. J Leukoc Biol 2008;83:1345-1353.

17 Bonvillain RW, Painter RG, Adams DE, Viswanathan A, Lanson NA Jr, Wang G: RNA interference against CFTR affects HL60-derived neutrophil microbicidal function. Free Radic Biol Med 2010;49:1872-1880.

18 Phennicie RT, Sullivan MJ, Singer JT, Yoder JA, Kim CH: Specific resistance to Pseudomonas aeruginosa infection in zebrafish is mediated by the cystic fibrosis transmembrane conductance regulator. Infect Immun 2010; 78:4542-4550.

19 Bonfield TL, Hodges CA, Cotton CU, Drumm ML: Absence of the cystic fibrosis transmembrane regulator (CFTR) from myeloid-derived cells slows resolution of inflammation and infection. J Leukoc Biol 2012;92:11111122.

20 Kutner RH, Zhang XY, Reiser J: Production, concentration and titration of pseudotyped HIV-1-based lentiviral vectors. Nat Protoc 2009;4:495-505.

21 Nauseef WM, McCormick SJ, Clark RA: Calreticulin functions as a molecular chaperone in the biosynthesis of myeloperoxidase. J Biol Chem 1995;270:4741-4747. 
-22 Moyer BD, Loffing J, Schwiebert EM, LoffingCueni D, Halpin PA, Karlson KH, Ismailov, II, Guggino WB, Langford GM, Stanton BA: Membrane trafficking of the cystic fibrosis gene product, cystic fibrosis transmembrane conductance regulator, tagged with green fluorescent protein in Madin-Darby canine kidney cells. J Biol Chem 1998;273:21759-21768.

-23 Granio O, Norez C, Ashbourne Excoffon KJ, Karp PH, Lusky M, Becq F, Boulanger P, Zabner J, Hong SS: Cellular localization and activity of Ad-delivered GFP-CFTR in airway epithelial and tracheal cells. Am J Respir Cell Mol Biol 2007;37:631-639.

24 Oceandy D, McMorran B, Schreiber R, Wainwright BJ, Kunzelmann K: GFP-tagged CFTR transgene is functional in the G551D cystic fibrosis mouse colon. J Membr Biol 2003;192: 159-167.

25 Robert R, Carlile GW, Liao J, Balghi H, Lesimple P, Liu N, Kus B, Rotin D, Wilke M, de Jonge HR, Scholte BJ, Thomas DY, Hanrahan JW: Correction of the delta Phe508 cystic fibrosis transmembrane conductance regulator trafficking defect by the bioavailable compound glafenine. Mol Pharmacol 2010;77: 922-930.

26 Klebanoff SJ: Myeloperoxidase: contribution to the microbicidal activity of intact leukocytes. Science 1970;169:1095-1097.

-27 Aratani Y, Koyama H, Nyui S, Suzuki K, Kura F, Maeda N: Severe impairment in early host defense against Candida albicans in mice deficient in myeloperoxidase. Infect Immun 1999;67:1828-1836.
28 Aratani $\mathrm{Y}$, Kura F, Watanabe H, Akagawa H, Takano Y, Ishida-Okawara A, Suzuki K, Maeda N, Koyama H: Contribution of the myeloperoxidase-dependent oxidative system to host defence against Cryptococcus neoformans. J Med Microbiol 2006;55:1291-1299.

29 Aratani Y, Kura F, Watanabe H, Akagawa H, Takano Y, Suzuki K, Maeda N, Koyama H: Differential host susceptibility to pulmonary infections with bacteria and fungi in mice deficient in myeloperoxidase. J Infect Dis 2000; 182:1276-1279.

30 Parry MF, Root RK, Metcalf JA, Delaney KK, Kaplow LS, Richar WJ: Myeloperoxidase deficiency: prevalence and clinical significance. Ann Intern Med 1981;95:293-301.

31 Koh AY, Priebe GP, Ray C, Van Rooijen N, Pier GB: Inescapable need for neutrophils as mediators of cellular innate immunity to acute Pseudomonas aeruginosa pneumonia. Infect Immun 2009;77:5300-5310.

32 Bonvillain RW, Painter RG, Ledet EM, Wang $\mathrm{G}$ : Comparisons of resistance of $\mathrm{CF}$ and nonCF pathogens to hydrogen peroxide and hypochlorous acid oxidants in vitro. BMC Microbiol 2011;11:112.

33 Segal AW, Coade SB: Kinetics of oxygen consumption by phagocytosing human neutrophils. Biochem Biophys Res Commun 1978; 84:611-617.
34 Winterbourn CC, Hampton MB, Livesey JH, Kettle AJ: Modeling the reactions of superoxide and myeloperoxidase in the neutrophil phagosome: implications for microbial killing. J Biol Chem 2006;281:39860-39869.

35 Painter RG, Wang G: Direct measurement of free chloride concentrations in the phagolysosomes of human neutrophils. Anal Chem 2006; 78:3133-3137.

36 Aiken ML, Painter RG, Zhou Y, Wang G: Chloride transport in functionally active phagosomes isolated from human neutrophils. Free Radic Biol Med 2012;53:2308-2317.

37 Denning GM, Anderson MP, Amara JF, Marshall J, Smith AE, Welsh MJ: Processing of mutant cystic fibrosis transmembrane conductance regulator is temperature-sensitive. Nature 1992;358:761-764.

- 38 Mall M, Kreda SM, Mengos A, Jensen TJ, Hirtz S, Seydewitz HH, Yankaskas J, Kunzelmann K, Riordan JR, Boucher RC: The DeltaF508 mutation results in loss of CFTR function and mature protein in native human $\mathrm{CO}^{-}$ lon. Gastroenterology 2004;126:32-41.

39 Haws CM, Nepomuceno IB, Krouse ME, Wakelee H, Law T, Xia Y, Nguyen H, Wine JJ: Delta F508-CFTR channels: kinetics, activation by forskolin, and potentiation by xanthines. Am J Physiol 1996;270:C1544-C1555.

40 Cholon DM, O’Neal WK, Randell SH, Riordan JR, Gentzsch M: Modulation of endocytic trafficking and apical stability of CFTR in primary human airway epithelial cultures. Am J Physiol Lung Cell Mol Physiol 2010. 298:L304-L314. 